\title{
O Inventário de Recursos do Ambiente Familiar
}

\author{
The Home Environment Resources Scale
}

\author{
Edna Maria Marturano* \\ Universidade de São Paulo, Ribeirão Preto, Brasil
}

\begin{abstract}
Resumo
O objetivo deste artigo é descrever o desenvolvimento do RAF - Inventário de Recursos do Ambiente Familiar. Baseado na concepção ecológica do desenvolvimento, o RAF avalia recursos do ambiente familiar que podem contribuir para o aprendizado acadêmico nos anos do ensino fundamental, em três domínios: recursos que promovem processos proximais; atividades que sinalizam estabilidade na vida familiar; práticas parentais que promovem a ligação família-escola. Uma revisão de pesquisas que utilizaram o RAF indica índices aceitáveis de consistência interna, bem como associação entre escores no RAF e indicadores de desempenho escolar e ajustamento. Passeios, brinquedos e livros, bem como oportunidades de interação com os pais em casa, foram recursos relacionados a indicadores de bom desempenho escolar e ajustamento. Embora ainda sejam necessários estudos de validação e fidedignidade, o inventário tem sido uma ferramenta útil para pesquisadores e pode ser usado por profissionais que trabalham em contexto clínico ou educacional.

Palavras-chave: Avaliação do ambiente familiar; desempenho escolar; instrumento de avaliação.

Abstract

The aim of this article is to describe the development of the HERS - Home Environment Resources Scale. The HERS is based on an ecological view of development. It assesses support resources available to the child at home, which can contribute for school achievement. These resources relate to three domains: resources that promote proximal processes; activities that signal stability in family processes; parental practices that promote a home-school linkage. Previous research using the HERS has indicated acceptable indexes of internal consistency, as well as significant associations between HERS scores and measures of academic achievement and adjustment. Leisure activities, toys, books, and opportunities for the child to interact with parents at home were all related to child achievement and adjustment. Although further studies are needed to secure its reliability and validity, the instrument has proven to be a useful tool for researchers, being used by professionals who work in clinical and educational contexts.

Keywords: Assessment of home environment; school achievement; assessment instrument.
\end{abstract}

Os pais e a família têm um papel importante na aprendizagem escolar das crianças. A partir dos anos 50 do século passado, observou-se um aumento de interesse dos pesquisadores em investigar influências da família no aprendizado escolar. A pesquisa nesse campo começou focalizando variáveis distais, como o nível sócio-econômico, porém foi a partir da década de 60 que os estudos ganharam impulso, quando se passou a investigar a influência de processos da vida familiar sobre o desempenho das crianças na escola (Kellaghan, Sloane, Alvarez \& Bloom, 1993).

Os resultados dessas pesquisas sugerem que os pais e a família podem direcionar positivamente $o$ aprendizado escolar, a motivação da criança para os estudos e o desenvolvimento de competências interpessoais que garantem um bom relacionamento com professores e colegas. Diversos aspectos da vida familiar são importantes, incluindo desde a atmosfera e organização do lar até o envolvimento direto dos pais com a vida escolar da criança (Bradley, Caldwell \& Rock, 1988a; Stevenson \& Baker, 1987).

\footnotetext{
"Endereço para correspondência: Faculdade de Medicina de Ribeirão Preto USP - Bloco da Saúde Mental. Rua Tenente Catão Roxo, 2.650. Ribeirão Preto, SP. 14051-140.E-mail emmartur@fmrp.usp.br. Apoio: $\mathrm{CNPq}$
}

A configuração de recursos que são relevantes para o desempenho muda à medida que a criança se desenvolve, mas os efeitos do ambiente familiar têm sido identificados nos diferentes níveis de ensino: na educação infantil, no ensino fundamental e médio e até mesmo na universidade (Kellaghan, 1977; King, 1998; Nelson, 1984; Reynolds, 1989; Wood, Chapin, \& Hannah, 1985). Da mesma forma, o envolvimento direto dos pais com a vida escolar dos filhos parece se um preditor significativo de progresso acadêmico, desde a meninice até o final da adolescência (Connors \& Epstein, 1995; Stevenson \& Baker, 1987).

Em que pese o interesse dos pesquisadores pela investigação do tema, faltam instrumentos com a finalidade específica de identificar, no âmbito da família, recursos promotores de desempenho escolar. Os autores empregam ora instrumentos desenvolvidos com outros objetivos, como o inventário HOME (Caldwell \& Bradley, 1984), ora questionários com um foco circunscrito, como por exemplo, o envolvimento dos pais na vida escolar da criança ou atividades de letramento no lar. Em um rastreamento de artigos empíricos indexados a partir de 1960, um único instrumento abrangente foi localizado, a Home Rating Scale (Christenson, 1990), um roteiro que avalia nove aspectos do 
ambiente do lar relevantes para o progresso escolar da criança. Mas a Home Rating Scale deve ser aplicada domiciliarmente, o que, se por um lado aumenta a validade ecológica das informações obtidas, por outro lado tem um elevado custo financeiro. Esse também é o caso do inventário HOME.

O objetivo deste artigo é descrever o desenvolvimento de um inventário para levantamento de recursos do ambiente familiar que podem contribuir para o aprendizado acadêmico nos anos do ensino fundamental. Sua construção decorreu das limitações expostas no parágrafo precedente, relativas a instrumentos disponíveis na literatura. Tendo sido concebido para finalidades de pesquisa e aplicação, espera-se que o RAF - Inventário de Recursos do Ambiente Familiar - possa ser útil à comunidade de pesquisadores $\mathrm{e}$ profissionais interessados no tema.

\section{Construção do RAF - Fundamentação Teórica e Empírica}

O estudo das condições que contribuem para o aprendizado escolar se ajusta ao modelo bioecológico do desenvolvimento (Bronfenbrenner, 1986, 1979/1996; Bronfenbrenner \& Morris, 1998). No estágio inicial de desenvolvimento do RAF, uma revisão da literatura visando identificar experiências específicas que contribuem para o desempenho e o ajustamento escolar (Marturano, 1998) indicou que os estudos revistos poderiam ser integrados nessa perspectiva, com ênfase nos conceitos de processos proximais, estabilidade ambiental, microssistema e mesossistema.

Os processos proximais são considerados os mecanismos primários do desenvolvimento humano. Bronfenbrenner e Morris (1998) os definem como "processos de interação recíproca e cada vez mais complexa entre um organismo humano ativo em desenvolvimento e as pessoas, objetos e símbolos em seus ambientes externos imediatos" (p. 996). O conceito de estabilidade ambiental está relacionado ao de processos proximais; os autores afirmam que ". . processos proximais não podem funcionar efetivamente em ambientes que são instáveis e imprevisíveis no espaço e no tempo" (p. 1019). A família e a escola são microssistemas onde ocorrem os processos proximais, constituindo um mesossistema de ligações e processos entre os dois sistemas, com efeitos sobre o desenvolvimento da criança (Bronfenbrenner, 1986).

Com base nesses conceitos, os resultados da revisão da literatura que precedeu a construção do RAF foram agrupados em três domínios amplos de recursos do ambiente familiar que podem contribuir para o progresso acadêmico. No nível do microssistema familiar, os recursos foram organizados em dois domínios: recursos que promovem processos proximais e atividades previsiveis que sinalizam algum grau de estabilidade na vida familiar. Recursos operando no nível do mesossistema família-escola foram identificados como práticas parentais que promovem a ligação família-escola.

O domínio recursos que promovem processos proximais compreende as seguintes áreas de recursos e atividades: participação em experiências estimuladoras do desenvolvimento, como passeios e viagens; oportunidades de interação com os pais; disponibilidade de brinquedos e materiais que apresentam desafio ao pensar; disponibilidade de livros, jornais e revistas; uso adequado do tempo livre; acesso a atividades programadas de aprendizagem (Bradley, 1993; Bradley et al., 1988a; Caldwell \& Bradley, 1984; Kellaghan et al., 1993; Siegel, 1990; Snow, Barnes, Chandler, Goodman \& Hemphill, 1990; Wang, Haertel \& Walberg, 1990). No domínio atividades previsiveis que sinalizam algum grau de estabilidade na vida familiar estão incluídas áreas de recurso tais como rotinas e reuniões regulares da família e cooperação da criança em tarefas domésticas (Bradley et al., 1988a; Caldwell \& Bradley, 1984; Kellaghan, 1977; Siegel, 1990; Snow et al., 1990).

As práticas parentais que promovem a ligação família-escola englobaram indicadores de envolvimento direto dos pais na vida escolar, como participação nas reuniões e acompanhamento das notas (Connors \& Epstein, 1995; García Bacete \& Rosel Ramirez, 200 1; Izzo, Weissberg, Kasprow, \& Fendrick, 1999; Kellaghan, 1977; Marjoribanks, 2001; Reynolds, 1989).

A construção do inventário se deu no contexto do atendimento a crianças com queixa escolar. A criação de itens representativos das áreas relevantes foi feita mediante levantamento em relatos de entrevistas conduzidas com mães / cuidadores de 70 crianças. Nesse levantamento, foram identificadas descrições de recursos disponíveis no ambiente familiar, apontados pela literatura como favorecedores do desempenho acadêmico. Com base nas descrições de recursos contidas nos relatos, foram elaboradas as perguntas abertas e os itens de escolha múltipla que compõem o instrumento. O roteiro foi pré-testado em amostra de 20 famílias, procedendo-se a mudanças na redação de alguns itens. (Marturano, 1999; Marturano, Alves \& Santa Maria, 1998).

A análise da primeira versão baseou-se na aplicação do RAF a 100 famílias de crianças com queixa escolar. Essa análise indicou que a participação em tarefas domésticas estava associada ao gênero, razão pela qual o tópico foi excluído do inventário a partir de então. (Marturano, Ferreira \& D’Avila-Bacarji, 2005).

\section{Aplicação e Correção}

A versão do RAF reproduzida no Anexo A compõe-se de dez tópicos, que correspondem às áreas de recursos identificadas previamente. Aplica-se o inventário sob forma de entrevista semi-estruturada, em que cada tópico é apresentado à mãe / informante oralmente, tendo o examinador liberdade para parafrasear o conteúdo da questão caso haja dificuldade de compreensão por parte da pessoa entrevistada. Em cada tópico, o entrevistador inicia fazendo a pergunta aberta que o introduz. Assinala os itens mencionados pela pessoa entrevistada em sua resposta livre e, em seguida, apresenta os demais itens, um a um. Se na resposta à pergunta inicial for informado um item que não consta da lista, este deve ser marcado e descrito no item "outro".

A pontuação bruta em cada um dos dez tópicos é a soma dos itens assinalados, com exceção dos tópicos 8, 9 e 10, que têm pontuação específica indicada. Para obter uma pontuação relativa, sugere-se a fórmula pontuação bruta/ pontuação má- 
xima do tópico $x 10$, onde a pontuação máxima corresponde ao número de itens, exceto nos tópicos 8,9 e 10. A pontuação relativa é útil para análises comparativas entre os tópicos.

Duas regras valem para o item "outro": (a) ele só será computado uma vez na pontuação bruta do tópico, mesmo quando a pessoa entrevistada mencionar mais de um recurso não presente na lista de itens ${ }^{1}$; (b) somente será incluído na pontuação máxima quando presente na pontuação bruta.

\section{Fidedignidade}

Pesquisas conduzidas com diferentes versões do RAF têm encontrado indícios aceitáveis de fidedignidade. D'Avila-Bacarji (2004), em uma amostra de 30 crianças com queixa escolar e 30 sem problemas, obteve um índice de consistência interna igual a 0,76 (alfa de Cronbach), baseado nos escores dos tópicos. Usando versão reduzida do instrumento, com sete tópicos, aplicada a mães de 70 crianças que estavam iniciando o ensino fundamental, TrivellatoFerreira (2005) encontrou um alfa igual a 0,70. Marturano et al. (2005) obtiveram a =0,84 com base nos escores dos itens, em amostra de 100 crianças com queixa escolar.

A estabilidade da medida global de recursos derivada do RAF foi investigada em um único estudo, e de forma restrita: Santos (1999) entrevistou três mães com intervalo de 20 dias, obtendo os seguintes percentuais de acordo entre as respostas das duas aplicações: 100, 99 e $92 \%$.

\section{Associação com desempenho escolar}

Evidências de associação entre medidas derivadas do RAF e indicadores de desempenho escolar têm sido obtidas no contex to clínico, focalizando crianças com queixa escolar, e também em amostras da comunidade.

Marturano (1999) conduziu uma investigação com famílias de 100 crianças cursando as três primeira séries do ensino fundamental, que apresentavam a queixa escolar. Nesse estudo, o escore total no RAF foi um preditor significativo do nível de elaboração da escrita, em um texto produzido livremente pela criança. Ao comparar dois subgrupos, constituídos, respectivamente, por crianças que em sua produção mostraram apenas uma intenção de escrita ou elaboraram frases articuladas, a autora verificou que as crianças com escrita mais elaborada viviam em ambientes com maior disponibilidade de brinquedos e livros.

O escore total no RAF discriminou um grupo de 30 crianças, com idade média de 9 anos e 6 meses, identificadas com a queixa escolar, avaliadas no contex to clínico, em relação a 30 crianças não identificadas, avaliadas em uma escola (D'Avila-Bacarji, Marturano \& Elias, 2005a). Os grupos foram balanceados quanto a gênero, idade e escolaridade dos pais. Os tópicos do RAF que diferenciaram significativamente os dois grupos foram aqueles relacionados a uso do tempo livre, passeios, atividades compartilhadas com os pais em casa, brinquedos e livros. Uma análise discriminante "stepwise"

\footnotetext{
Essa regra não parece afetar restritivamente o escore de um tópico, visto que na aplicação da primeira versão a 100 famílias, nenhum item da categoria "outros" apresentou mais de um recurso.
}

sobre os dados desse estudo, tendo como variáveis independentes os escores dos tópicos, indicou $83 \%$ de precisão na discriminação dos grupos, sendo significativas para a correta classificação dos casos as medidas de atividades compartilhadas com os pais em casa e brinquedos.

Quando o grupo de crianças não identificadas com queixa escolar foi subdividido de acordo com a classificação no TDE - Teste de Desempenho Escolar de Stein (1994), o RAF discriminou os três grupos na direção esperada. Verificou-se que as crianças avaliadas na escola, com desempenho médio ou superior no TDE, eram as que tinham acesso a mais recursos no ambiente familiar, ao passo que as crianças com queixa escolar, avaliadas na clínica, viviam nos ambientes com menos recursos, ficando em posição intermediária as crianças sem queixa escolar, porém com desempenho inferior no TDE (D'Avila-Bacarji, Marturano \& Elias, 2005b). Praticamente os mesmos tópicos do estudo anterior continuaram diferenciando os grupos, com exceção do uso do tempo livre.

Moreira e Sigolo (2005a, 2005b), estudando o ambiente familiar de 14 alunos de escola pública na $4^{\mathrm{a}}$ série do ensino fundamental, verificaram que as crianças com alto rendimento escolar, quando comparadas aos colegas com baixo rendimento, envolviam-se mais em atividades com os pais e tinham mais acesso a passeios, atividades programadas e brinquedos.

Em seu estudo prospectivo com crianças que estavam iniciando a primeira série em escolas públicas, TrivellatoFerreira (2005) usou um modelo de regressão para o desempenho escolar que testou, como possíveis preditores, cinco medidas obtidas no início do ano letivo, relativas a características da criança e do ambiente familiar: nível intelectual, habilidade de detectar rimas, recursos do ambiente (RAF), relacionamento pai-criança harmonioso e práticas parentais positivas. $\mathrm{O}$ modelo final indicou como preditores significativos de desempenho a habilidade de detectar rimas, o escore no RAF e a medida de práticas parentais positivas, respondendo, em conjunto, por $45 \%$ da variância nos escores brutos do TDE. O melhor preditor foi a habilidade da criança em detectar rimas $(\mathrm{R} 2=0,29)$, seguindo-se o escore no $\operatorname{RAF}(\mathrm{R} 2=0,10)$ e a medida de práticas parentais positivas $(\mathrm{R} 2=0,06)$. Essa autora obteve também uma correlação positiva significante entre a pontuação no RAF e a avaliação posterior do desempenho escolar das crianças, feita pelos professores $(\mathrm{r}=0,33)$.

Os dados da pesquisa de Trivellato-Ferreira (2005) foram retomados por Marturano et al. (2005), que calcularam correlações entre o escore total, os escores nos tópicos do RAF e a pontuação das crianças nos subtestes de leitura, escrita e aritmética do TDE. O escore total do RAF correlacionou significativamente com a pontuação em todos os subtestes do TDE (leitura $=0,47$; escrita $=0,48$; aritmética $=0,54)$. Com relação aos tópicos, cinco mostraram associação estatisticamente significante com algum indicador de desempenho. Os escores relativos a passeios e brinquedos mostraram correlação positiva significativa com o desempenho nos três subtestes do TDE; a disponibilidade de livros correlacionou com o desempenho em escrita e aritmética; 
o envolvimento direto dos pais na vida escolar e a disponibilidade de jornais e revistas correlacionaram com o desempenho em leitura. As correlações significativas variaram entre 0,54 (brinquedos $\mathrm{x}$ aritmética) e 0,24 (livros $\mathrm{x}$ escrita).

Associação com indicadores de ajustamento.

Tendo sido concebido para identificar recursos promotores de desempenho escolar, o RAF foi usado também na investigação de características do ambiente familiar associadas a indicadores de ajustamento. Os resultados dessas pesquisas são relevantes para uma análise do instrumento, visto que a ação do ambiente familiar sobre o desempenho se dá por múltiplas vias, inclusive a do ajustamento da criança às demandas do contexto escolar (Johnson, Cowan \& Cowan, 1999; Marturano, 1998).

Ferreira e Marturano (2002) investigaram a associação entre variáveis do ambiente familiar e problemas de comportamento em 67 crianças com queixa escolar atendidas em uma clínica de psicologia. As crianças cursavam predominantemente as três primeiras séries do ensino fundamental (97\%) e suas idades variavam entre sete e 11 anos. Comparando dois grupos formados com base na avaliação de problemas de comportamento pelas mães, encontraram diferenças significativas, favorecendo o grupo sem problemas de comportamento, no escore total e nos seguintes tópicos do RAF: passeios, atividades compartilhadas com os pais em casa, brinquedos.

A questão da associação entre recursos do ambiente familiar e indicadores de ajustamento foi retomada nos estudos de Ferriolli (2005) e Trivellato-Ferreira (2005). A primeira conduziu uma investigação junto a um Núcleo de Atenção Primária e Saúde da Família, com o objetivo de detectar mecanismos de risco e proteção associados a problemas emocionais / comportamentais em crianças de 6 a 12 anos. Com base na avaliação de problemas de comportamento feita pelas mães, em uma amostra de 100 famílias, conduziu análises de regressão logística univariada e multivariada, tendo como variável dependente o risco associado aos problemas de comportamento, e como variáveis independentes os escores nos tópicos do RAF, expressos em totais de pontos, e medidas de adversidade ambiental, como stress materno e eventos de vida adversos. $\mathrm{Na}$ análise multivariada, identificou duas ordens de recursos avaliadas pelo RAF, que operam em processos de proteção frente ao risco de transtornos emocionais ou de comportamento: uso do tempo livre e rotinas com horário definido (Ferriolli, 2005).

Enquanto os estudos de Ferreira e Marturano (2002) e Ferriolli (2005) focalizaram problemas de comportamento e se basearam em avaliações da criança feitas pelas mães, Trivellato-Ferreira (2005) usou indicadores de comportamento socialmente adequado, com base na percepção dos professores. Obteve correlação positiva significante entre uma pontuação global de recursos derivada do RAF e medidas de competência social $(r=0,40)$ e ajustamento em sala de aula $(\mathrm{r}=0,37)$.

Marturano et al. (2005) retomaram os dados de Trivellato-Ferreira (2005) e calcularam correlações entre os escores nos tópicos do RAF e a medida de competência social. Encontraram correlação positiva entre competência social e os seguintes indicadores de recursos do ambiente familiar: brinquedos $(r=0,37)$, passeios $(r=0,35)$, atividades compartilhadas com os pais em casa $(\mathrm{r}=0,25)$.

\section{Outras informações disponíveis sobre o RAF}

No momento em que este artigo foi escrito, tínhamos conhecimento de pesquisas utilizando o RAF para caracterizar o ambiente familiar de crianças com necessidades especiais (Pamplin, 2005), convivendo com mães depressivas (Mian, 2005), e expostas à violência intrafamiliar (Grossi, 2005). Um estudo empregou o inventário para explorar efeitos do ambiente familiar sobre a eficácia de uma intervenção destinada a atenuar as dificuldades acadêmicas e comportamentais de crianças com queixa escolar (Giurlani, 2004).

O estudo de Pamplin (2005) sobre inclusão de crianças com necessidades educacionais especiais focalizou as famílias de 61 crianças que freqüentavam salas municipais de recurso, além do ensino regular. A autora comparou dois grupos, com base na categoria de sala de recurso que a criança freqüentava: deficiência ou fracasso escolar. Observou que as crianças freqüentando salas para fracasso escolar tinham menos acesso a recursos no ambiente familiar, notadamente passeios, atividades programadas, brinquedos, livros, revistas e reuniões regulares da família.

Dois estudos de comparação de grupo investigaram recursos do ambiente familiar de crianças vivendo em situação de risco psicossocial. No ambiente familiar de crianças expostas à depressão materna foi observada menor presença de recursos promotores do desenvolvimento, menor oportunidade de interação com os pais e menor organização da rotina (Mian, 2005). Já no estudo com crianças expostas à violência doméstica, os indicadores de recursos não diferiram entre os grupos (Grossi, 2005).

Giurlani (2004) acompanhou 30 meninos com queixa escolar (idade média 9 anos e 3 meses, escolaridade entre $1^{\text {a }} \mathrm{e}$ $4^{a}$ série). Avaliando o desempenho e o comportamento das crianças antes e depois de uma intervenção que combinava o treino em solução de problemas interpessoais com atividades psicopedagógicas, verificou que o grupo com melhoras mais acentuadas no TDE tinha mais acesso a passeios, atividades compartilhadas com os pais em casa e brinquedos. Por outro lado, de acordo com a avaliação das mães, o grupo de crianças cujos problemas de comportamento persistiram tinha mais acesso a passeios. Neste caso, provavelmente uma segunda variável estava envolvida, já que este grupo também apresentava maior exposição à instabilidade financeira (Giurlani, 2004).

Outros resultados de pesquisas indicam correlações positivas entre medidas globais derivadas do RAF e uso de convenções na escrita e ortografia (Santos, 1999); escolaridade materna (Marturano, 1999); qualidade do relacionamento pai-criança, número de pessoas na rede de suporte à mãe, nível intelectual e habilidade da criança para detectar rimas (Trivellato-Ferreira, 2005). 


\section{Concluindo: Limites e Possibilidades no Uso do RAF}

O Inventário de Recursos do Ambiente Familiar foi construído sobre três pilares - uma concepção ecológica do desenvolvimento, um levantamento de evidências de pesquisa relacionando o desempenho acadêmico aos recursos disponíveis no contexto familiar e uma ampla sondagem junto a famílias de crianças com queixa escolar, seja diretamente, seja através da consulta a relatos de entrevistas. As informações reunidas neste artigo, decorrentes de pesquisas que empregaram o instrumento com diferentes propósitos, sugerem que o RAF pode oferecer indicadores válidos de recursos que favorecem o desempenho acadêmico nos anos do ensino fundamental.

O escore total no RAF mostrou associação significativa com desempenho em todas as investigações que focalizaram essa associação, apesar da diversidade entre os estudos em vários aspectos: a associação foi detectada entre diferentes medidas de desempenho escolar e diferentes composições de tópicos para obtenção do escore total do RAF; em estudos seja exploratórios, comparativos ou preditivos; com grupos de crianças em distintas condições de aprendizagem; em modelos de regressão que incluíram outros preditores de desempenho escolar, além do RAF (D'AvilaBacarji et al., 2005a, 2005b; Marturano, 1999; Marturano et al., 2005; Santos, 1999; Trivellato-Ferreira, 2005).

A investigação de Trivellato-Ferreira (2005) sugere que o escore total no RAF constitui um indicador global de recursos promotores do desenvolvimento em sentido amplo, e não apenas de suporte ao aprendizado escolar e ajustamento na sala de aula. Essa autora encontrou correlações entre o RAF e medidas de nível intelectual, habilidade de detectar rimas e competência social.

As correlações encontradas nos diversos estudos, entre o escore total no RAF e indicadores de desempenho e ajustamento da criança, são apenas moderadas. Variando entre 0,33 e 0,54, elas se aproximam dos resultados obtidos com o inventário HOME, um conhecido instrumento de avaliação baseado em entrevista e observação direta do ambiente familiar (Caldwell \& Bradley, 1984). Em dois estudos, respectivamente com 42 e 124 crianças, o escore total na versão elementar do HOME (para crianças de 6 a 10 anos) mostrou correlações com indicadores de desempenho, que variaram entre 0,37 e 0,41, e com medidas de ajustamento, entre 0,30 e 0,50 (Bradley et al., 1988; Bradley, Caldwell, Rock, Hamrick \& Pandia, 1988b).

As correlações modestas entre escores do RAF e medidas de desempenho poderiam ser interpretadas como indícios de fragilidade do instrumento. Por outro lado, pode-se supor que elas estejam mascarando relações complexas entre processos da vida familiar e o desempenho acadêmico das crianças. Os resultados da investigação de Trivellato-Ferreira (2005) com escolares iniciantes ilustram essa possibilidade: a habilidade da criança para detectar rimas foi melhor preditor de desempenho escolar que o escore total do RAF, mas manteve correlação significativa com a medida do RAF, embora em grau de colinearidade aceitável para a regressão. Esses resultados sugerem que a influência dos recursos ambientais sobre o desempenho acadêmico inicial seria em parte mediada pelas capacidades linguísticas da criança, uma hipótese que pode ser verificada empiricamente.

Em concordância com o esquema conceitual usado para organizar os domínios de recursos do ambiente familiar no RAF, as variáveis vistas como recursos promotores de processos proximais foram as que mostraram associação mais consistente com resultados escolares. O conjunto de recursos mais significativos inclui o acesso a passeios, a disponibilidade de livros e brinquedos, a oportunidade de interação com os pais em casa. Ao passo que a disponibilidade de livros se apresenta como recurso específico, favorecedor de desempenho escolar, os passeios e brinquedos se mostram como recursos promotores de desenvolvimento em sentido amplo, correlacionando com outras capacidades cognitivas da criança, relacionadas à inteligência, à linguagem e à competência social. No geral, essas relações são consistentes com pesquisas anteriores (Bradley, 1993; Bradley et al., 1988a; Caldwell \& Bradley, 1984; Kellaghan et al., 1993; Siegel, 1990; Snow et al., 1990; Wang et al., 1990).

A oportunidade de interação com os pais em casa também aparece como um conjunto amplo de recursos, associados a bons resultados acadêmicos e comportamentais da criança. De acordo com a literatura, interações com os pais constituem processos proximais dos mais significativos para o desenvolvimento afetivo e interpessoal, lingüístico e cognitivo (Kellaghan et al., 1993; Marturano, 1998; Sigel, Stinson \& Flaugher, 1991; Snow et al., 1990).

A generalidade dos efeitos positivos do acesso a passeios, brinquedos e interações com os pais, do modo como são avaliados no RAF, foi apontada também no estudo de Giurlani (2004), onde tais recursos se mostraram associados aos efeitos positivos de uma intervenção psicopedagógica, incidindo sobre o desempenho escolar.

Ao passo que em diversos estudos os indicadores de recursos promotores de processos proximais foram associados a melhores resultados acadêmicos, as medidas de atividades previsiveis que sinalizam algum grau de estabilidade na vida familiar não mostraram associação com desempenho escolar. Esses resultados poderiam ser um argumento contra a manutenção dos tópicos referentes a rotinas e reuniões da família. No entanto, parece importante manter esses tópicos para detectar aqueles lares caóticos, nos quais a desorganização da vida cotidiana pode afetar indiretamente o rendimento acadêmico, por interferir, por exemplo, na saúde emocional da criança. Alguns estudos utilizando o RAF obtiveram resultados que apontam nessa direção. Na pesquisa de Ferriolli (2005) com famílias cadastradas no Programa de Saúde da Família, rotinas com horário definido apareceram como um fator de proteção frente ao risco de transtornos emocionais ou de comportamento. Mian (2005) verificou que em famílias com mães depressivas havia menos rotinas regulares, quando comparadas a famílias com mães sem diagnóstico psiquiátrico. Ferreira e Marturano (2002) verificaram que crianças com problemas de comportamento associado à queixa escolar tinham menos horários definidos para brincar e fazer a lição de casa, quando com- 
paradas às crianças que apresentavam apenas a queixa escolar. Atraso escolar de pelo menos dois anos, em relação ao esperado para a idade, apareceu associado à ausência de horário para levantar-se de manhã, jantar e fazer a lição de casa (Marturano, 1999).

Com relação aos recursos operando no nível do mesossistema família-escola, contemplado em um único tópico no RAF, o indicador de práticas parentais que promovem a ligação família-escola mostrou apenas uma correlação significativa moderada com leitura $(r=0,27)$, em crianças que estavam ingressando no ensino fundamental (Marturano et al., 2005). Nos demais estudos, cujas amostras incluíam crianças mais velhas, com desempenho escolar pobre, não se detectou relação entre esse tópico e os indicadores de desempenho. Esses resultados à primeira vista são incompatíveis com a literatura recente, que enfatiza a importância do envolvimento parental para o progresso acadêmico das crianças (Connors \& Epstein, 1995; Izzo et al., 1999; Marjoribanks, 2001). Entretanto, eles podem ser explicados, em parte, pela presença de crianças identificadas com a queixa escolar, em todos os estudos que não encontraram associação entre envolvimento parental e desempenho, já que os pais tendem a se envolver mais com a vida acadêmica dos filhos quando estes apresentam um desempenho insatisfatório, notadamente com crianças de segunda a quinta série (Watkins, 1997).

Embora os resultados dos estudos conduzidos até então com o RAF apresentem indícios de fidedignidade e validade aceitáveis para este instrumento, é importante indicar algumas de suas limitações. Em primeiro lugar, ele não oferece uma cobertura exaustiva dos recursos familiares favorecedores do progresso acadêmico, mas apenas ajuda a identificar algumas áreas mais representativas. Como exemplo de processos familiares não contemplados no RAF podem-se citar as expectativas dos pais em relação ao desempenho escolar de seus filhos. Uma segunda limitação a ser lembrada é que, por ter sido construído com um foco em crianças com queixa escolar, provavelmente o RAF discrimina melhor os ambientes pouco estimuladores, sendo menos sensível para discriminar ambientes enriquecidos. Nesse sentido, é plausível supor que o elenco de itens representativos das áreas relevantes seria mais diversificado se o levantamento de recursos tivesse sido feito, não mediante entrevistas com cuidadores de crianças que apresentavam a queixa escolar, mas com cuidadores de crianças apresentando desempenho acadêmico excelente. Em terceiro lugar, deve-se assinalar que as informações disponíveis sobre o RAF ainda são esparsas, havendo apenas um estudo sistemático focalizando propriedades psicométricas do instrumento (Marturano et al., 2005). Como esse trabalho se apoiou em amostras pequenas para os padrões de estudos psicométricos, são necessários estudos confirmatórios com amostras maiores. Por fim, o impacto do fator socioeconômico nos resultados do RAF ainda não foi adequadamente dimensionado. Esta é uma limitação importante em instrumentos de avaliação de recursos ambientais. Um estudo com o HOME elementar indicou correlação de 0,53 com a escolaridade materna e 0,58 com um indicador de nível socioeconômico (Bradley et al., 1988a). No único artigo que focalizou essa questão em relação ao RAF, Marturano (1999) encontrou correlação positiva entre o escore total e escolaridade materna $(0,34)$; encontrou também correlações significativas entre um índice de bens de consumo disponíveis no lar e escores nos tópicos brinquedos $(0,61)$, livros $(0,44)$, passeios $(0,39)$ e rotinas com horário definido $(0,35)$. Embora a questão requeira mais ampla investigação, os estudos de D'Avila-Bacarji et al. (2005a, 2005b) sugerem que o instrumento mantém sua sensibilidade quando se comparam grupos homogêneos quanto ao indicador socioeconômico representado pela escolaridade do casal parental.

Levando-se em conta esses limites do instrumento, o RAF pode ser considerado uma ferramenta útil não apenas para pesquisa, mas também para aplicação, em contex to clínico ou educacional. O formato de entrevista, se por um lado não oferece um panorama tão rico de detalhes como a observação in loco, por outro lado ganha em praticidade, economia e facilidade de aplicação, o que torna o instrumento acessível para uso em diferentes contextos. O procedimento de entrevista semi-estruturada favorece um rastreamento abrangente, ao mesmo tempo em que permite a investigação de particularidades. Informações obtidas junto às famílias através do RAF podem subsidiar programas de apoio aos pais de crianças com dificuldades escolares, visando motivar e ajudar os pais a otimizarem recursos já disponíveis no contex to familiar, de modo a melhorar a qualidade do apoio ao desenvolvimento dos filhos.

\section{Referências}

Bradley, R. H. (1993). Children's home environments, health, behavior, and intervention efforts: A review using the HOME inventory as a marker measure. Genetic, Social, and General Psychology Monographs, 119, 439-490.

Bradley, R. H., Caldwell, B. M., \& Rock, S. L. (1988a). Home environment and school performance: A ten-year follow-up and examination of three models of environmental action. Child Development, 59, 852-867.

Bradley, R. H., Caldwell, B. M., Rock, S. L., Hamrick, H. M., \& Pandia, H. (1988b). Home observation for measurement of the environment: Development of a home inventory for use with families having children 6 to 10 years old. Contemporary Educational Psychology, 13, 58-71.

Bronfenbrenner, U. (1986). Ecology of the family as a context for human development: research perspectives. Developmental Psychology, 6, 723-742.

Bronfenbrenner, U. (1996). A ecologia do desenvolvimento humano: Experimentos naturais e planejados (M. A. V. Veronesi, Trad.). Porto Alegre, RS: Artes Médicas. (Original publicado em 1979)

Bronfenbrenner, U., \& Morris, P. (1998). The ecology of developmental processes. In W. Damon \& R. M. Lerner (Eds.), Handbook of child Psychology: Vol. 1. Theoretical models of human development (pp. 993-1028). New York: John Wiley.

Caldwell, B. M., \& Bradley, R. H. (1984). Home observation for measurement of the environment. Little Rock, AR: University of Arkansas at Little Rock.

Connors, L. J., \& Epstein, J. L. (1995). Parent and school partnerships. In M. A. Bornstein (Ed.), Handbook of parenting: Vol. 4. Applied and practical parenting (pp. 437-458). Mahwah, NJ: Lawrence Erlbaum. 
Christenson, S. L. (1990). Difference: In students home environments: the need to work with families. School Psychology Review, 19, 505-5 17

D’Avila-Bacarji, K. M. G. D. (2004). O ambiente familiar de crianças referidas para atendimento psicológico por dificuldades escolares: Um estudo comparativo com alunos não referidos. Dissertação de Mestrado não-publicada, Programa de Pós-Graduação em Saúde Mental, Universidade de São Paulo, Ribeirão Preto, SP.

D’Avila-Bacarji, K. M. G., Marturano, E. M., \& Elias, L. C. S. (2005a). Suporte parental: Um estudo sobre crianças com queixas escolares. Psicologia em Estudo, 10, 107-115.

D’Avila-Bacarji, K. M. G., Marturano, E. M., \& Elias, L. C. S. (2005b). Recursos e adversidades no ambiente familiar de crianças com desempenho escolar pobre. Paidéia: Cadernos de Psicologia e Educação, 15, 43-55.

Ferreira, M. C. T., \& Marturano, E. M. (2002). Ambiente familiar e os problemas de comportamento apresentados por crianças com baixo desempenho escolar. Psicologia: Reflexão e Crítica, 15, 35-44.

Ferriolli, S. H. T. (2005). Identificação de condições de risco para transtornos emocionais e de comportamento em crianças na fase escolar cadastradas em um Núcleo de Atenção Primária e Saúde da Família. Texto de Exame de Qualificação não-publicado, Programa de Pós-Graduação em Saúde Mental, Universidade de São Paulo, Ribeirão Preto, SP.

García Bacete, F. J., \& Rosel Ramirez, J. (2001). Family and personal correlates of academic achievement. Psychological Reports, 88, 533-547.

Giurlani, A. G. (2004). Ambiente familiar e o efeito do Programa EPRP destinado a atenuar problemas de comportamento e aprendizagem. Dissertação de Mestrado não-publicada, Programa de Pós-Graduação em Saúde Mental, Universidade de São Paulo, Ribeirão Preto, SP.

Grossi, R. (2005). Crianças em risco psicossocial: Percurso de vida pós-ação do Conselho Tutelar. Texto de Exame de Qualificação não-publicado, Programa de Pós-Graduação em Saúde Mental, Universidade de São Paulo, Ribeirão Preto, SP.

Izzo, C. V., Weissberg, R. P., Kasprow, W. J., \& Fendrich, M. (1999). A logitudinal assessment of teacher perceptions of parent involvement in children's education and school performance. American Journal of Community Psychology, 27, 817-839.

Johnson, V., Cowan, P. A., \& Cowan, C. P. (1999). Children's classroom behavior- The unique contribution of family organization. Journal of Family Psychology, 13, 355-371.

Kellaghan, T. (1977). Relationship between home environment and scholastic behavior in a disadvantaged population. Journal of Educational Psychology, 69, 754-760.

Kellaghan, T., Sloane, K., Alvarez, B., \& Bloom, B. S. (1993). The home environment and school learning: Promoting parental involvement in the education of children. San Francisco: Jossey-Bass.

King, A. K. (1998). Family Environment Scale predictors of academic performance. Psychological Reports, 83, 13 19-1327.

Marjoribanks, K. (2001). Family and ability correlates of academic achievement. Psychological Reports, 89, $510-512$.

Marturano, E. M. (1998). Ambiente familiar e aprendizagem escolar. In C. A. Funayama (Ed.), Problemas de aprendizagem: Enfoque multidisciplinar (pp. 73-90). Ribeirão Preto, SP: Legis Summa.

Marturano, E. M. (1999). Recursos no ambiente familiar e dificuldades de aprendizagem na escola. Psicologia: Teoria e Pesquisa, 15, 135-142.

Marturano, E. M., Alves, M. V., \& Santa Maria, M. R. (1998). Recursos do ambiente familiar e desempenho na escola. In A. W. Zuardi, E. M. Marturano, M. A. C. Figueiredo \& S. R. Loureiro (Eds.), Estudos em Saúde Mental - 1998 (pp. 48-77). Ribeirão Preto, SP: Curso de Pós-Graduação em Saúde Mental da Faculdade de Medicina de Ribeirão Preto.
Marturano, E. M., Ferreira, M. C. T., \& D’Avila-Bacarji, K. M. G. (2005). An evaluation scale of family environment for the identification of children at risk of school failure. Psychological Reports, 96, 307-321.

Mian, L. (2005). Comportamento, autopercepções e ambiente familiar de crianças que convivem com mães com história de depressão. Dissertação de Mestrado não-publicada, Programa de Pós-Graduação em Saúde Mental, Universidade de São Paulo, Ribeirão Preto, SP.

Moreira, G. M., \& Sigolo, S. R. R. L. (2005a). As relações de influências do ambiente familiar e do contexto escolar na história pregressa de crianças que estão no final do I ciclo do ensino fundamental [Resumo]. In D. D. Dell'Aglio (Ed.), Anais V Congresso Brasileiro de Psicologia do Desenvolvimento (pp. 218-219). São Paulo, SP: SBPD.

Moreira, G. M., \& Sigolo, S. R. R. L. (2005b). Recursos na história pregressa de crianças que estão na $4{ }^{a}$ série do ensino fundamental [Resumo]. In Associação Nacional de Pesquisa em Psicologia Escolar e Educacional (Ed.), Anais Eletrônicos Encontro de Pesquisa em Educação no Brasil/Região Sudeste - Educação: Direito ou Serviço? [CD-ROM] (p. 7). Belo Horizonte, MG: ANPED.

Nelson, G. (1984). The relationship between dimensions of classroom and family environments and the self-concept, satisfaction, and achievement of grade 7 and 8 students. Journal of Consulting Psychology, 12, 276-287.

Pamplin, R. (2005). A interface família-escola na inclusão de crianças com necessidades educacionais especiais: Uma perspectiva ecológica. Dissertação de Mestrado não-publicada, Programa de Pós-Graduação em Educação Especial, Universidade Federal de São Carlos, SP.

Reynolds, A. (1989). A structural model of first-grade outcomes for an urban, low socioeconomic status, minority population. Journal of Educational Psychology, 81, 594-603.

Santos, L. C. (1999). Crianças com dificuldade de aprendizagem: Estudo de seguimento. Dissertação de Mestrado não-publicada, Programa de Pós-Graduação em Saúde Mental, Universidade de São Paulo, Ribeirão Preto, SP.

Siegel, D. F. (1990). The literacy press: A process model for reading development. Journal of Educational Research, 83, 336-347.

Sigel, I., Stinson, E. T., \& Flaugher, J. (1991). Socialization of representational competence in the family: the distancing paradigm. In L. Okagaki \& R. J. Sternberg (Eds.), On the development of children thinking (pp. 121-144). Hillsdale, NJ: Erlbaum.

Snow, C. E., Barnes, W. S., Chandler, J., Goodman, I. F., \& Hemphill, L. (1990). Unfulfilled expectations: Home and school influences on literacy. Cambridge, MA: Harvard University Press.

Stein, L. M. (1994). TDE: Teste de Desempenho Escolar: Manual para aplicação e interpretação. São Paulo, SP: Casa do Psicólogo.

Stevenson, D. J., \& Baker, D. P. (1987). The family-school relation and the child's school performance. Child Developement, 58, 1348-1357.

Trivellato-Ferreira, M. C. (2005). As tarefas de desenvolvimento da meninice e a transição para o ensino fundamental. Tese de Doutorado não-publicada, Programa de Pós-Graduação em Psicologia, Universidade de São Paulo, Ribeirão Preto, SP.

Wang, M. C., Haertel, G. D., \& Walberg, H. J. (1990). What influences learning? A content analysis of review literature. Journal of Educational Research, 84, 30-43.

Watkins, T. J. (1997) Teacher communications, child achievement, and parent traits in parent involvement models. The Journal of Educational Research, 91, 3-13.

Wood, J., Chapin, K., \& Hannah, M. E. (1985). Family environment and its relationship to underachievement. Adolescence, 23, 283-290. 


\section{Anexo A}

\section{RAF - INVENTÁRIO DE RECURSOS DO AMBIENTE FAMILIAR}

Aplica-se o roteiro sob forma de entrevista semiestruturada, em que cada tópico é apresentado à mãe / informante oralmente, tendo o examinador liberdade para parafrasear o conteúdo da questão caso haja dificuldade de compreensão por parte da pessoa entrevistada. Em cada tópico, o entrevistador inicia fazendo a pergunta aberta que o introduz. Após registrar a resposta a essa pergunta, apresenta, uma a uma, as demais alternativas de resposta.

1. O QUE A CRIANÇA FAZ QUANDO NÃO ESTÁ NA
ESCOLA?
$\square$ Assiste à TV
$\square$ Ouve rádio
$\square$ Joga video-game
$\square$ Lê livros, revistas, gibis
$\square$ Brinca na rua
$\square$ Brinca dentro de casa
$\square$ Outro - especificar

2. QUAIS OS PASSEIOS QUE A CRIANÇA REALIZOU NOS ÚLTIMOS 12 MESES?

$$
\text { Passeio }
$$

Bosque Municipal

Evento anual da cidade (feira, rodeio, ...)

口 Cinema ou teatro

Lanchonete

口 Praia

Viagem de trem

- Sítio, chácara ou fazenda

Centro da cidade

Museu

Aeroporto

Outros - especificar:

\section{HÁ ATIVIDADES PROGRAMADAS QUE A CRIANÇA REALIZA REGULARMENTE?}

[ Faz catecismo, estudos bíblicos ou evangelização.

口 Freqüenta núcleo municipal do bairro

- Pratica esporte em clubes, academias, ginásios.

口 Freqüenta aulas para aprender atividade artesanal (por ex.emplo: tapeçaria, pintura...).

- Tem aulas de piano, violão ou outro instrumento musical.

口 Freqüenta algum programa de atividades para crianças, como o Kurumim

口 Tem aulas de inglês ou outro idioma

口 Faz computação

Outro - especificar

\section{QUAIS AS ATIVIDADES QUE OS PAIS DESENVOL- VEM COM A CRIANÇA EM CASA?}

Brincar

Jogar video-game ou outros jogos

A Assistir a filmes

Assistir a programas infantis na TV

Contar estórias e casos

Ler livros, revistas

- Conversar sobre como foi o dia na escola

- Conversar sobre notícias, filmes e outros programas de TV

- Ouvir as estórias da criança; conversar sobre os assuntos que ela traz

- Realizar juntos atividades domésticas, como: lavar o carro, fazer almoço ou outras

$\square$ Outras - especificar

5. QUAIS OS BRINQUEDOS QUE ELE (ELA) TEM OU

JÁ TEVE? SEU FILHO TEM OU JÁ TEVE:

$\square$ uma cama só para ele

brinquedos de andar ( triciclo, bicicleta, patinete... )

brinquedos para movimentos corpo (corda de pular, balanço...)

口 instrumento musical de brinquedo ou de verdade(tambor, pianinho...)

- brinquedo que lida com números ( dados, dominó... )

$\square$ brinquedos de letras (abecedários, quebra -cabeças com letras...)

$\square$ brinquedo de aprender cores, tamanhos, formas ( quebracabeça, encaixes. )

$\square$ brinquedos para conhecer nomes de animais (livros, miniaturas ...)

objetos como giz, lousa, cola, tinta, tesoura, lápis de cor e papel

aparelho de som com discos

um animal de estimação

livrinhos de estórias infantis

口 jogos de regras (dama, loto, senha, memória...)

brinquedos de faz de conta ( panelinhas, bonecas, martelo, serrote ...)

brinquedos de construção ( blocos, lego, pinos mágicos )

$\square$ brinquedos de rodas (carrinhos, trens, carrinho de boneca ...)

$\square$ videogame

$\square$ bola, pipa, bola de gude, carrinho rolemã

$\square$ outros, especifique

\section{HÁ JORNAIS E REVISTAS NA SUA CASA?}

não

sim - tipo:

$\square$ jornal

$\square$ revista - $\square$ de notícias - $\square$ de TV - $\square$ feminina - $\square$ de fotonovela - $\square$ de esporte - $\square$ religiosa - 0 outra, especifique 


\section{HÁ LIVROS NA SUA CASA?}

não

$\square$ sim - tipo:

$\square$ escolares

$\square$ romances, contos, literatura

livrinhos infantis

religiosos (bíblia, evangelhos, catecismo)

$\square$ técnicos, científicos

$\square$ enciclopédias

$\square$ dicionário

$\square$ outros, especifique

\section{ALGUÉM EM CASA ACOMPANHA A CRIANÇA NOS AFAZERES DA ESCOLA?}

Alguém em casa: ninguém a mãe o pai outra pessoa

Verifica se $o$

material escolar

está em ordem

Avisa quando é

hora de ir para

a escola

Supervisiona a

lição de casa

Supervisiona o

estudo para as

provas

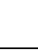

$\square$

Comparece às reuniões da escola

Acompanha as notas e a freqüência às aulas

Pontuação de cada item: mãe e pai $=3$; só a mãe $=2$, só o pai $=2$; outra pessoa $=1 ;$ mãe $/$ pai e outra pessoa $=1 ;$ ninguém $=0$.
9. SEU FILHO TEM HORA CERTA PARA:

\begin{tabular}{lccc} 
& sempre & às vezes & nunca \\
\hline almoçar & $\square$ & $\square$ & $\square$ \\
tomar banho & $\square$ & $\square$ & $\square$ \\
brincar & $\square$ & $\square$ & $\square$ \\
ir dormir & $\square$ & $\square$ & $\square$ \\
levantar-se de manhã & $\square$ & $\square$ & $\square$ \\
jantar & $\square$ & $\square$ & $\square$ \\
fazer a lição de casa & $\square$ & $\square$ & $\square$ \\
assistir à TV & $\square$ & $\square$ & $\square$ \\
\hline
\end{tabular}

Pontuação: sempre $=2$; às vezes $=1$; nunca $=0$.

10. SUA FAMÍLIA COSTUMA ESTAR REUNIDA:

\begin{tabular}{lccc} 
& sempre & às vezes & nunca \\
\hline no café da manhã & $\square$ & $\square$ & $\square$ \\
no almoço & $\square$ & $\square$ & $\square$ \\
no jantar & $\square$ & $\square$ & $\square$ \\
à noite, para assistir à TV & $\square$ & $\square$ & $\square$ \\
\hline
\end{tabular}

e nos fins de semana :

em casa $\quad \square \quad \square \quad \square$ em passeios

口

Pontuação: sempre $=2$; às vezes $=1 ;$ nunca $=0$. 